\title{
Health service organisation impact on lower extremity amputations in people with type 2 diabetes with foot ulcers: systematic review and meta-analysis
}

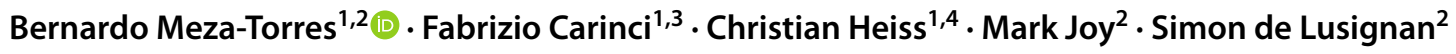

Received: 12 October 2020 / Accepted: 17 December 2020 / Published online: 6 February 2021

(c) The Author(s) 2021

\begin{abstract}
Aims Despite the evidence available on the epidemiology of diabetic foot ulcers and associated complications, it is not clear how specific organizational aspects of health care systems can positively affect their clinical trajectory. We aim to evaluate the impact of organizational aspects of care on lower extremity amputation rates among people with type 2 diabetes affected by foot ulcers.

Methods We conducted a systematic review of the scientific literature published between 1999 and 2019, using the following key terms as search criteria: people with type 2 diabetes, diagnosed with diabetic foot ulcer, treated with specific processes and care pathways, and LEA as primary outcome. Overall results were reported as pooled odds ratios and $95 \%$ confidence intervals obtained using fixed and random effects models.

Results A total of 57 studies were found eligible, highlighting the following arrangements: dedicated teams, care pathways and protocols, multidisciplinary teams, and combined interventions. Among them, seven studies qualified for a meta-analysis. According to the random effects model, interventions including any of the four arrangements were associated with a $29 \%$ reduced risk of any type of lower extremity amputation $(\mathrm{OR}=0.71 ; 95 \% \mathrm{CI} 0.52-0.96)$. The effect was larger when focusing on major LEAs alone, leading to a $48 \%$ risk reduction ( $\mathrm{OR}=0.52 ; 95 \% \mathrm{CI} 0.30-0.91)$.

Conclusions Specific organizational arrangements including multidisciplinary teams and care pathways can prevent half of the amputations in people with diabetes and foot ulcers. Further studies using standardized criteria are needed to investigate the cost-effectiveness to facilitate wider implementation of improved organizational arrangements. Similarly, research should identify specific roadblocks to translating evidence into action. These may be structures and processes at the health system level, e.g. availability of professionals with the right skillset, reimbursement mechanisms, and clear organizational intervention implementation guidelines.
\end{abstract}

Keywords Type 2 diabetes · Diabetic foot ulcers · Health service organization · Lower extremity amputation

\section{Introduction}

Managed By Massimo Porta.

Bernardo Meza-Torres

bernardo.meza-torres@phc.ox.ac.uk

1 Department of Clinical and Experimental Medicine, University of Surrey, Guildford, UK

2 Nuffield Department of Primary Care Health Sciences, University of Oxford, Oxford, UK

3 Department of Statistical Sciences, University of Bologna, Bologna, Italy

4 Surrey and Sussex Healthcare NHS Trust, East Surrey Hospital, Redhill, UK
Lower extremity amputations (LEA) represent one of the most challenging complications experienced by people with type 2 diabetes (T2D). However, recent studies show a reduction of major amputations, this has been attributed to improved quality of health care provided over the last 15 years. Still, this contrasts with minor amputations, which in recent years have shown rates with stagnant declines or even slight increases, broadening the scope to improve outcomes [1-3].

Diabetes-related foot ulcers (DFU) are associated with higher rates of complications, in particular, renal failure, LEA, and disability. It has been shown that only between 50 and $60 \%$ of people affected by DFU survive more than 5 
years after their first diagnosis $[4,5]$. The cost of DFUs is estimated as one-third of the total costs of diabetes-related treatment $[4,6]$.

Despite the evidence available on the epidemiology of foot ulcers and associated complications [7-9], it is not clear how specific organizational aspects of health care systems can positively affect the trajectory between the first diagnosis of DFU and LEA.

In this study, we aimed to conduct a systematic review investigating the effectiveness of processes of care following the initial presentation of DFUs, as measured by the change in the rate of LEA over time.

This paper aims to respond to the following research questions:

- Which organizational factors are associated with reduced LEA rates following initial diagnosis of DFU in patients with type 2 diabetes?

- Which of these organizational arrangements can be pooled in a meta-analysis?

\section{Materials and methods}

The population in included studies were adults diagnosed with T2D who presented with a foot ulcer as an index event. The occurrence of any lower extremity amputation was the primary endpoint. Major amputations were considered separately as a clinically relevant endpoint.

We conducted a systematic search on July 2019 using PubMed Medline and Excerpta Medica database (EMBASE), targeting studies published between 1999 and 2019. The search comprised four, main semantic components: (1) patients with diabetes, (2) with diabetic foot ulcer, (3) in the context of processes and care pathways, and (4) experiencing LEA or ulcer healing as potential negative/positive outcomes.

The search strings used in each database have been combined as follows: "Diabetes Mellitus" [MeSH] AND ("Diabetic Foot"[Mesh] OR (diabet*[tiab]) OR "Foot Ulcer"[Mesh] OR (foot ulcer*[tiab]) OR Leg Ulcer [MeSH]) AND ((process*OR pathway*) AND care) AND ("Amputation"[Mesh] OR (amputation*[tiab]) OR (Lower extremity amputation*[tiab]) OR (LEA[tiab])).

To be eligible, each study had to include an organizational arrangement as a specific intervention of interest, considered as the main exposure.

To help interpretation, we classified the identified organizational arrangements according to the Donabedian model for evaluating quality of care [10]. This framework for health systems research indicates how modifying the structures and processes at the healthcare system or provider level can predict patient-level outcomes. Thus, this study considered prevention strategies implemented at the care provider level.
Therefore, patient-level interventions alone were not considered, e.g. standalone patient education strategy.

Our classification was drawn from the approach adopted by recognized institutions, including the TRIAD Study Group [11, 12], the UK's National Institute for Health and Care Excellence [13-15], the International Consortium for Health Outcomes Measurement [16], the International Working Group on the Diabetic Foot [17], and various authors' view on integrated care $[5,18]$.

We created the following categories of organizational arrangements:

- Dedicated teams: involving the implementation of human and structural resources, e.g. specialty nurses and dedicated inpatient beds, to specifically treat diabetic foot ulcers.

- Multidisciplinary teams: involving an integrated framework of multispecialty care, whose resources might not be exclusive to DFU. They are coordinated to manage DFU through a care plan, a key worker/manager, and joint activities, e.g. foot clinics integrating diabetology, podiatry, specialty nurses, vascular/orthopaedic surgery, microbiology, wound care, nutrition, etc.

- Care pathways: including explicit and dedicated step-bystep protocols for the local care team to treat a patient in a specific setting, e.g. risk profiling, access to prevention and treatment, referencing systems, and a clinical setting for chronic disease monitoring.

- Combined interventions: complex interventions integrating the above elements without a predominant one. Since only arrangements implemented at the level of care providers were considered, the presence or absence of patient-level interventions implemented alongside was not considered for this categorization, e.g. patient education interventions.

The literature review was conducted according to the guidelines of the Preferred Reporting Items for Systematic Reviews and Meta-Analyses (PRISMA) [19] Flow and the Cochrane Handbook for Systematic Reviews of Interventions v.5.1. [20].

We considered as eligible study designs all controlled or observational studies, either prospective or retrospective, as well as systematic reviews or meta-analyses. Narrative reviews, clinical practice guidelines, case series, case reports, or letters to editors were excluded from the search.

\section{Classification of eligible studies}

The full text of the selected papers was retrieved and assessed for eligibility by co-authors BMT and FC independently. Study eligibility for qualitative review was based on the following characteristics: (a) participants (people with 
T2D presenting with a foot ulcer as an index condition); (b) intervention (specific organizational arrangements); and (c) outcome (LEA as primary endpoint). The percentage of agreement was assessed using Cohen's kappa statistics [20].

Once selected, studies were stratified by BMT according to the type of quantitative outcome reported (e.g. binary outcome, OR, and RR), and by the category of organizational arrangements (e.g. dedicated teams, care pathways, multidisciplinary teams, and combined interventions). The list of references included in the identified studies was also examined for the inclusion of additional studies. Their eligibility for binary outcome meta-analysis was based on the following criteria: (a) studies presenting results in terms of quantitative measures; (b) quantitative measures must report the number of LEA cases as well as the number of persons at risk (population as denominator, not as rate); (c) and the quantitative measures should be reported for both an intervention and a control group (either in parallel or before vs after). The data collection form is included as supplement 1.

The risk of bias assessment was conducted on all studies finally selected, aided by the Newcastle-Ottawa Scale for cohort and case-control studies and the Cochrane Collaboration's tool for those studies with the respective designs $[21,22]$. Publication bias was assessed through funnel plot asymmetry analysis [20].

Quantitative assessment was carried out using a fixed and random effects meta-analytical approach for studies presenting results in terms of quantitative measures, e.g. the number of cases and number of people at risk separately, for both intervention and control groups. Overall results were calculated using a Mantel Hansel approach, weighted by the inverse variance [20]. We based our presentation of key results via fixed or random effects models, based upon the significance of the Q test of heterogeneity.

All statistical analyses were performed using the statistical package R v.1.2 and the metafor package [23, 24].

\section{Results}

As shown in Fig. 1, a total of $N=536$ unique studies were initially identified from Medline and Embase, based on the above-specified search strategy. Following further examination, a total of 142 articles were retrieved for full-text review, of which further 90 were excluded due to unrelated exposure $(N=32)$, unrelated outcome $(N=14)$, ineligible study types $(N=39)$, and other reasons $(N=5)$. Additionally, five studies were added by cross-referencing the retrieved studies. At the end of the process, a final subset of 57 articles were selected for review in the present article. The percentage of agreement between authors was of $56 \%$. The different organizational factors investigated in the final set of papers, along with the type of evidence supporting each of them, are shown in cross-tabulation in Table 1. The individual studies were graded as 'strong' when their findings were reported quantitatively, and as 'weak' when only narrative or non-significant quantitative results were reported. Due to heterogeneity in study designs, a single quality assessment tool could not be used to compare amongst the 57 studies. Hence an independent assessment by two authors was used.

The studies investigated the following organizational factors: care pathways, dedicated teams, multidisciplinary teams, and combined interventions.

Among the 57 studies identified, a total of $N=41(72 \%)$ reported an intervention that could be either categorized as a care pathway or combined intervention (combining care pathways with other interventions at the care provider level). Most combined interventions included care pathways coupled to multidisciplinary teams where specialists-in primary or secondary care-were integrated with a multidisciplinary setting. In some cases, combined interventions also included educational strategies in the outpatient setting.

Twenty of the studies (35\%) focused on interventions classified as care pathways, characterized by a protocol including multiple specialists that were not articulated within the same setting. Among them, $N=9$ (16\%) reported strong evidence of improvement. The remaining 11 studies (19\%) either showed weak support, including narrative argumentation and non-significant results, or in just two papers no evidence of an effect of care pathways on the reduction of LEA. A justification given for the lack of effect was that pathways may be complex to implement as they traverse organizational structures, creating barriers in the patient's journey.

Twenty-one papers (37\%) focused on the effect of combined interventions. Among them, four papers reported a statistically significant reduction in LEA rates, while ten addressed combined interventions including approaches of patient-centred care. In this subgroup of papers, one study found a lack of response of octogenarians with PAD to total amputation rates, independently from improvements in care and education.

A total of $N=15$ studies (18\%) reported the effect of multidisciplinary teams, all of them showing improvements. In some cases, it was shown that the multidisciplinary approach may be associated with increased workload, making the case for further cost-effectiveness research. However, these studies showed a remarkable heterogeneity in the composition of their teams, many involving primary and secondary care specialists (podiatry, nurses, vascular surgeons, and diabetologists) who were coordinated in the same physical space on certain days.

Dedicated teams were found only in one study, showing no statistical significance, but suggesting that podiatry services can help reducing LEA rates in selected high-risk subgroups. 


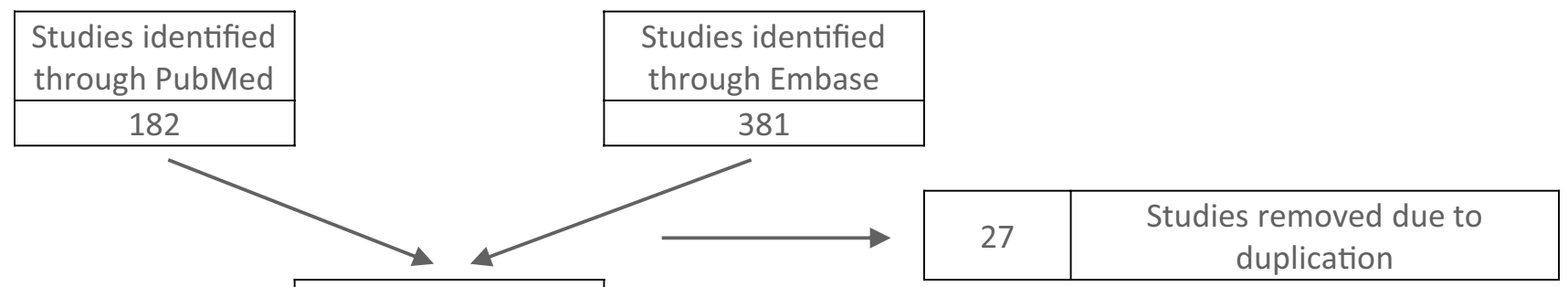

$\begin{gathered}\text { Unique studies } \\ \text { identified for title } \\ \text { nd abstract review }\end{gathered}$
536

$\longrightarrow$\begin{tabular}{|c|l|}
\hline 394 & Abstracts excluded with reasons \\
\hline 81 & Non-interested outcome \\
\hline 120 & Non-interested exposure \\
\hline 101 & Ineligible study types \\
\hline 40 & Off-topic \\
\cline { 1 - 2 } 30 & No results \\
\cline { 1 - 2 } 22 & other \\
\hline
\end{tabular}

\begin{tabular}{|c|}
\hline $\begin{array}{c}\text { Studies identified for } \\
\text { full-text review }\end{array}$ \\
\hline 142 \\
\hline
\end{tabular}

$\longrightarrow$\begin{tabular}{|c|l|}
\hline 90 & Full-texts excluded with reasons \\
\hline 32 & Non-interested exposure \\
\hline 14 & Non-interested outcome \\
\hline 39 & Ineligible study types \\
\hline 5 & Other \\
\hline
\end{tabular}

Studies eligible for

inclusion to systematic review

52

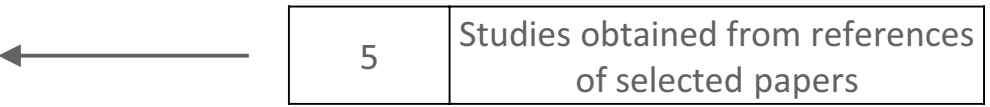

Fig. 1 Diagram of selection of eligible studies

Only seven of the studies were included in the metaanalysis [25-31], as they reported the number of cases of LEA as well as the population denominator (not as rate) for both the intervention and control groups (either in parallel or before vs after). The results obtained from the meta-analysis are presented in Fig. 2. The majority of these studies investigated combined interventions $(N=4)$, while the others focused on care pathways $(N=2)$ and multidisciplinary 
Table 1 Classification of results obtained from the literature review

\begin{tabular}{lllllc}
\hline Evidence & Care pathways & $\begin{array}{l}\text { Combined } \\
\text { intervention }\end{array}$ & Dedicated teams & $\begin{array}{l}\text { Multidis- } \\
\text { ciplinary } \\
\text { teams }\end{array}$ & Total \\
\hline 1. Strong against & - & $\mathbf{1}(5 \%)$ & - & - & $\mathbf{1}(2 \%)$ \\
2. Weak against & $\mathbf{2}(10 \%)$ & $\mathbf{1}(5 \%)$ & - & - & $\mathbf{3}(5 \%)$ \\
3. Inconclusive evidence & - & $\mathbf{5}(24 \%)$ & - & - & $\mathbf{5}(9 \%)$ \\
4. Weak support & $\mathbf{9}(45 \%)$ & $\mathbf{1 0}(48 \%)$ & $\mathbf{1}(100 \%)$ & $\mathbf{6}(40 \%)$ & $\mathbf{2 6}(46 \%)$ \\
5. Strong support & $\mathbf{9}(45 \%)$ & $\mathbf{4}(19 \%)$ & & $\mathbf{9}(60 \%)$ & $\mathbf{2 2}(39 \%)$ \\
Total & $\mathbf{2 0}(100 \%)$ & $\mathbf{2 1}(100 \%)$ & $\mathbf{1}(100 \%)$ & $\mathbf{1 5}(100 \%)$ & $\mathbf{5 7}(100 \%)$ \\
\hline
\end{tabular}

$N$ (percentage)

'Weak': supporting evidence with low statistical significance, or narrative support

'Strong': supporting evidence with statistical significance

\section{Intervention (n) Control (n)}

Author(s) and Year Events Total Events Total

\section{Relative \\ Weight \\ Risk Ratio \\ $[95 \% \mathrm{Cl}]$}

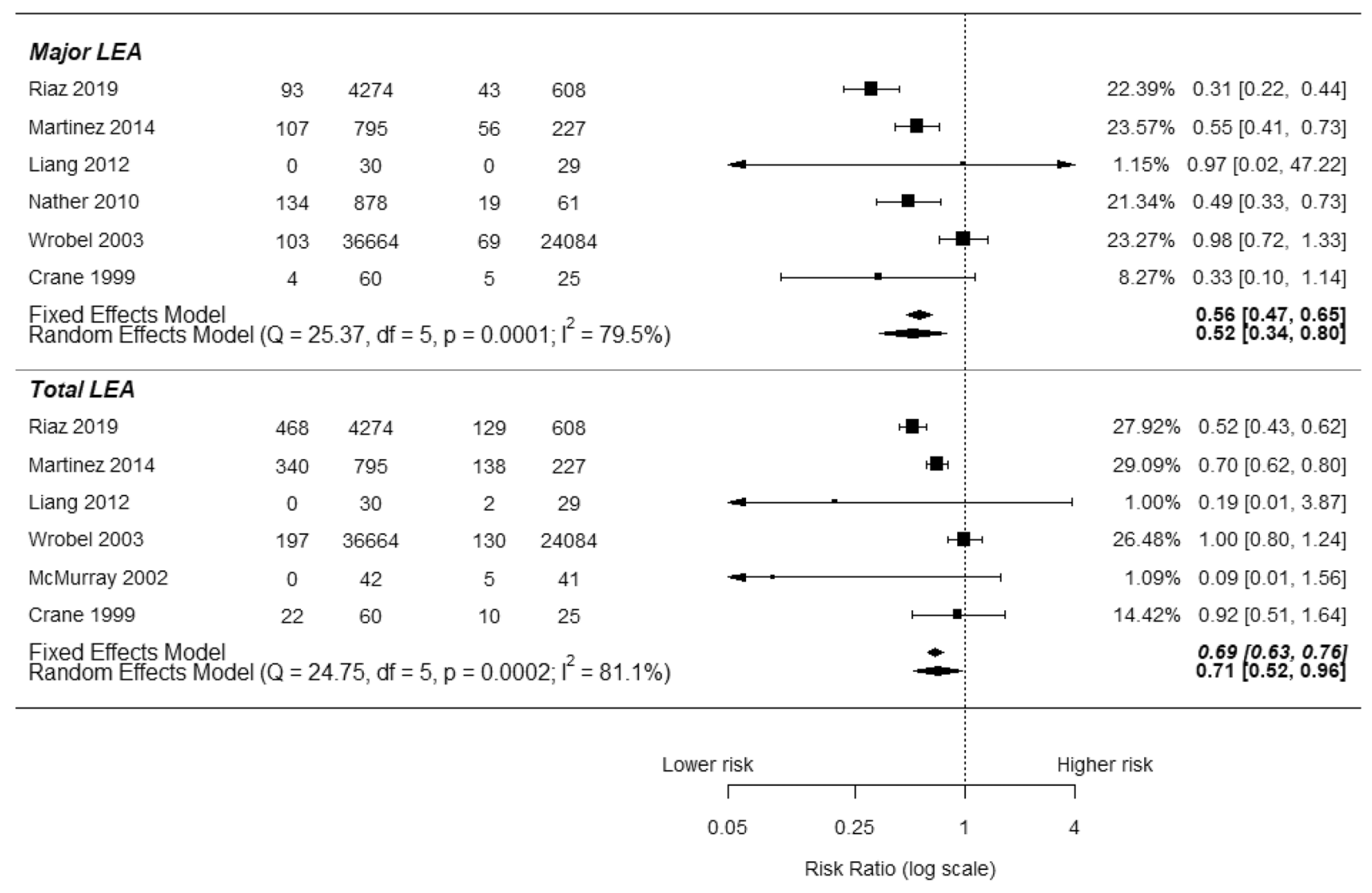

Fig. 2 Forest plot of quantitative meta-analysis of eligible studies

teams (see Table 2). Given the limited number of studies, we could not report results by type of organizational arrangement, but only in terms of organizational intervention yes/ no.

Overall, using the fixed model, we found that interventions including the four types of organizational arrangements were associated with a $31 \%$ reduced risk of any type of LEAs (OR $=0.69 ; 95 \%$ CI 0.63-0.76). Focusing on major LEAs alone, the result was equal to a $44 \%$ risk reduction (OR=0.56; 0.47-0.65).

However, the result of the $\mathrm{Q}$ heterogeneity test was highly significant in both cases $(p<0.001)$. 


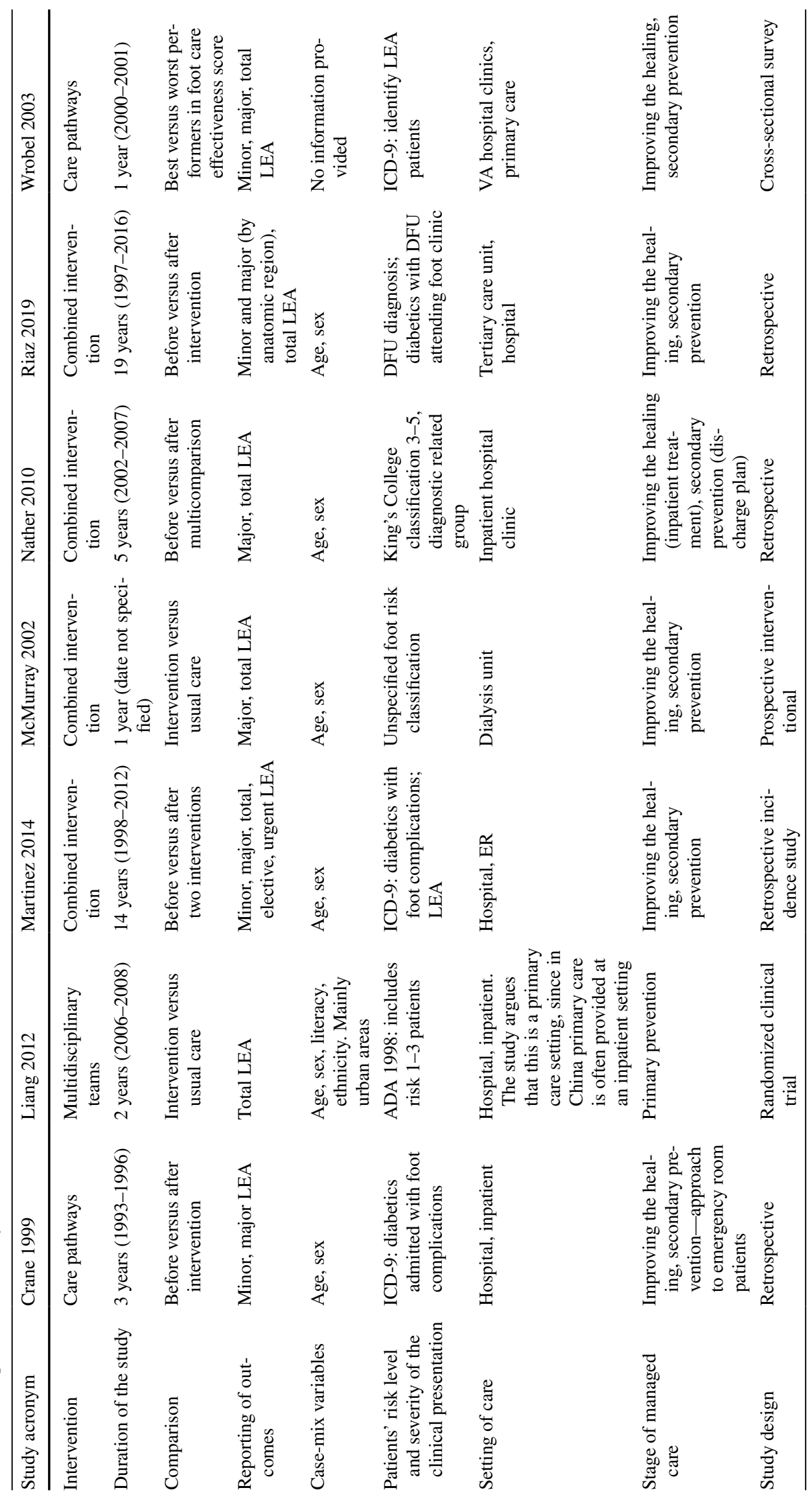




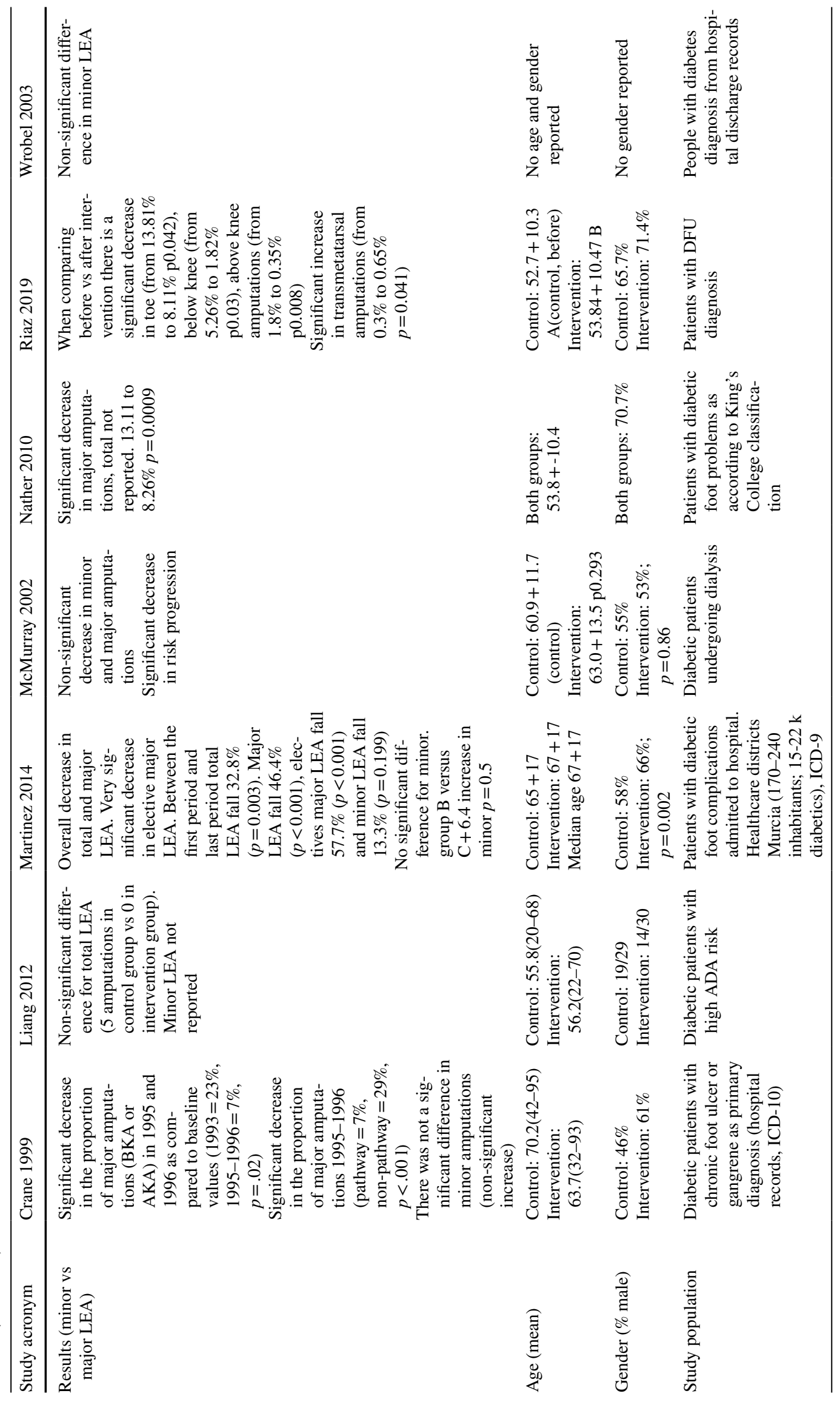




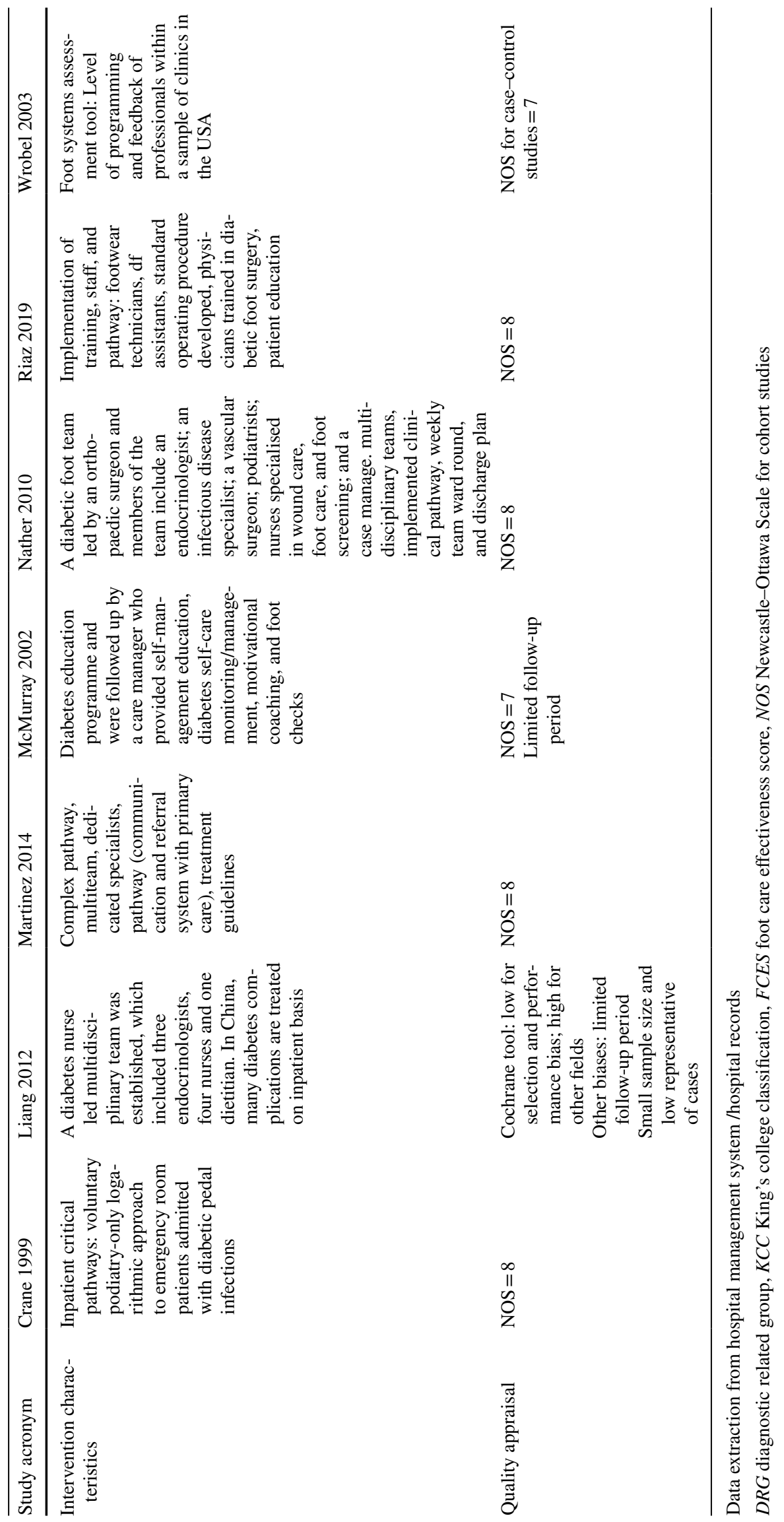


Therefore, we applied the random effects model as a more conservative estimate, showing a $29 \%$ risk reduction of any type of LEAs (OR $=0.71 ; 95 \%$ CI $0.52-0.96)$ and a larger effect on major LEAs alone, equal to a $48 \%$ risk reduction $(\mathrm{OR}=0.52 ; 95 \%$ CI $0.30-0.91)$.

Four studies [25-28] reported a decrease in the number of major LEAs while at the same time reporting either no difference or a slight increase in minor LEAs. Since data in these studies were aggregated, it was not possible to discern whether the increase in minor LEAs was due to multiple sequential minor amputations on the same patients.

Two of the studies with smaller sample sizes [30, 31] did not show statistically significant results (Fig. 2). Their small sample size is reflected in their lower quality scores (Table 2).

The above observation is consistent with publication bias funnel plots (Fig. 3), which show small-study asymmetry on left lower quadrant for the Total LEA subgroup, accounted by these studies. Regression tests for asymmetry analysis were not significant $(p>0.2)$ which is expected due to the low number of studies $(n<10)$ [20].

\section{Discussion}

Our systematic literature review identified fifty-seven eligible studies that have investigated the efficacy of different organizational factors in the management of people with diabetes and ulcers, namely care pathways, dedicated or multidisciplinary teams, and combined interventions. Overall, the majority of studies supported that these organizational factors can improve the outcome of patients with diabetes and ulcers.

\section{Main findings and generalizability}

Despite evidence-based guidelines for the prevention and treatment of diabetes and foot ulcers [32, 33], amputations and mortality are still high. Studies suggest that this is in part because guidelines for recommended management (e.g. multidisciplinary care) are not implemented and also low adherence of patient to treatments. Integrated care pathways are intended to tackle these problems by breaking guidelines down into simple steps and assigning clear responsibilities to different specialties that should be involved. For instance, foot care is an integral part of the NICE diabetes pathway [13] and includes essential elements aimed at reducing the risk of developing and
Fig. 3 Publication bias funnel plots
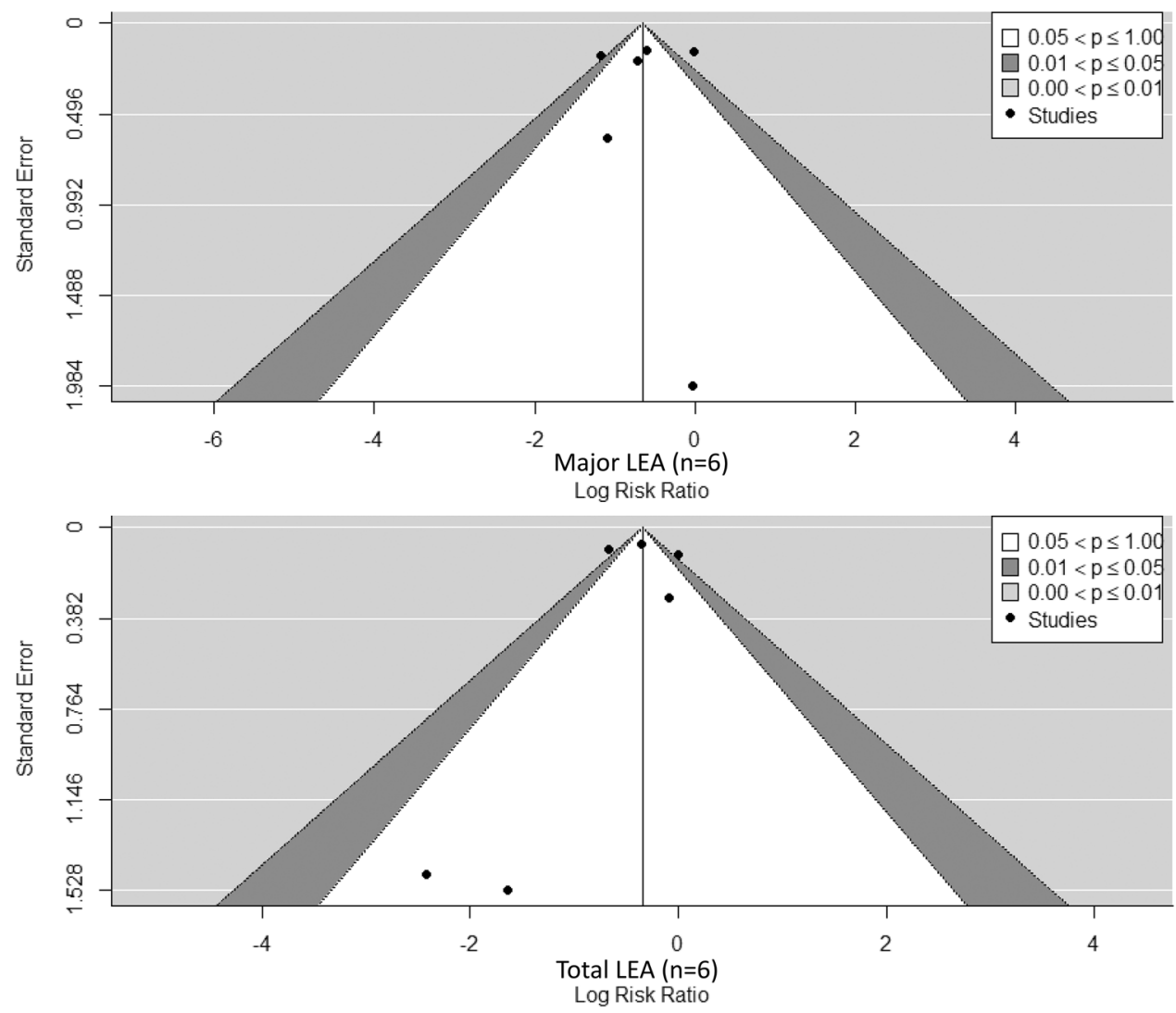
managing a diabetic foot problem. The majority of studies we identified showed that care pathways had a positive effect. Despite their heterogeneity, pathways shared an explicit and simpler depiction of the referral and treatment steps that are feasible under the already existing macrolevel structures and processes of care (e.g. reimbursement mechanisms, practice guidelines), or by implementing feasible changes at the local level structures-processes of care.

People with diabetes and ulcers generally have multimorbidities associated with the vascular complications of diabetes. Therefore, effective treatment is diverse, requiring expertise across several medical and surgical specialties. Guidelines require that the effective management of ulcers includes a multidisciplinary team [32-34]. Our current analysis supports this. The team compositions were heterogeneous (see Table 2) and it remains to be determined if this approach is cost-effective and which team members or training requirements are essential. Vascular medicine has been proposed as an effective building block of such a team [35]. This, however, can only be further investigated on a background of comparable care pathways.

Our search also showed that combined interventions may be effective. While care pathways were a component of all combined studies, several included multidisciplinary teams and patient-based interventions. Since there may exist a definition overlap with different arrangements of integrated care, combined interventions are here useful to depict the natural development of either intervention type into an increasingly complex one, in terms of categories, breadth, and degree[18, 36-38]. But this complexity may also make combined interventions harder to implement as a first step in clinical settings. Similarly, their evaluation may face multiple confounding factors.

Finally, the scarcity of studies on dedicated teams (only one) can be explained by the fact that healthcare professionals may not exclusively be dedicated to attending DFUs but looking also after other conditions.

Regarding our second research question, we found that among the fifty-seven studies identified, only seven could match the criteria for inclusion in a quantitative meta-analysis.

These studies demonstrated that specific organizational arrangements were significantly associated with a reduction of major LEAs. However, our current meta-analysis showed that organized care can prevent almost half the major amputations. Previous cost-effectiveness studies show that cost-intensive interventions that prevent $50 \%$ of amputations result in net savings of \$3000-4000 USD per patient [39]. The cost-saving impact could therefore be further investigated, considering that countries like England spend 0.9\% of the National Health Service budget on the management of DFU and LEAs [40].
There was a different mix of study designs, namely: retrospective observational, prospective observational, and crosssectional studies. Among them, observational studies had a longer duration and larger sample sizes. Therefore, their likelihood of reporting significant results was higher, with a larger magnitude of the effect that could be affected by bias $[26,27,29]$. Also, some observational designs reflect reallife data derived from electronic medical records (EMRs). This is important considering that experimental designs are hampered by the low incidence of LEA and long follow-up periods. There was also a mix in the study setting and stage of managed care. Observational studies more frequently included secondary care centres, e.g. hospital, emergency departments, and were more focused on secondary prevention, e.g. control of reulceration in patients already being treated for DFU. This is supported by the literature, where it has been argued that secondary prevention is plausible to be investigated because of the high 12-month reulceration rates (40\%) among selected populations, such as those presenting with DFU complications requiring specialist care [5].

Among case-mix variables, age and sex were consistently reported in all except one study (Wrobel 2003). They homogenously report an average age $>50$ years and male predominance. Other relevant demographic variables for case-mix adjustment, e.g. race or deprivation, were unreported, limiting population heterogeneity assessment and comparisons between studies.

In terms of DFU severity at baseline, only two studies reported a risk classification, e.g. King College Classification IV [29] and ADA 1998 [30]. All other studies only reported that patients had an established DFU diagnosis based on clinical codes. Ulcer grading is known to be linked to the outcome $[5,41]$ and should be considered for risk adjustment.

\section{Clinical relevance}

Four studies described the use of care pathways specifically introduced for the study intervention, while three reported to follow just care protocols recommended by general guidelines. Multidisciplinary teams in each study also evaluated a unique combination of professionals, varying in the content, duration, and intensity of the intervention. Nonetheless, common elements across all studies included the presence of podiatrists, specialized nurses, and glucose control performed by endocrinologists or primary care physicians. Relevant heterogeneity included a variable involvement of surgical specialists, e.g. vascular or orthopaedic surgeons, and a different complexity of care pathways, e.g. communication and referral systems, discharge plans, and dedicated attention days.

Studies also presented variation in target outcomes. Four studies [25-27, 29] reported significant reduction of major 
amputations, associated with either an increase or a nonsignificant decrease in minor amputations. Due to the limitations of study reporting, it was not possible to assess the impact of patient profiles, e.g. multiple minor amputations performed on the same patient.

Actionability of the results emerged from this study should also be seen in the perspective of the future organizational evolution of diabetes care after the emergency of the COVID-19 pandemic, which took place well after this study was conceived and conducted. The disruption of care delivery may translate into an increase in LEA rates [42]. Furthermore, evidence is emerging that people with T2D are at a higher risk of serious complications due to COVID-19 [43], potentially presenting an opportunity to regard coordinated remote consultations for preventing infections in the household and primary care centers, while enhancing opportunities for multidisciplinary consultation.

\section{Relevance for future studies}

The availability of structured healthcare databases using EMRs for surveillance [44] and quality of care monitoring [45] enables the construction of longitudinal cohorts on which clinical prediction models can be accurately validated. This approach can substantially reduce the cost of ad-hoc studies, while adjusting for relevant case-mix characteristics applying the Donabedian model considering both the individual and organizational levels. Moreover, the organization of standardized diabetes registers in an increasing number of countries can efficiently expand the approach to international comparisons of best practices.

\section{Methodological considerations}

Concerning the publication bias funnel plots (Fig. 3), they showed small-study asymmetry on the left lower quadrant for the total LEA subgroup, accountable to two studies with lower quality scores. This suggests a potential publication bias for total LEA, where no small studies reporting negative results were found, and where small-study publications may potentially report overestimated results. On the contrary, major LEA remains unaffected by this publication bias. Sufficiently large sample sizes are required to report enough LEA cases to disaggregate results into major-minor LEA, and larger publications are likely to get published regardless of the magnitude of the outcome. This may explain why small observational studies were not found for this subgroup.

The percentage of agreement between authors was lowmoderate $(56 \%)$. This can be attributed to the variability in the definition of the interventions of interest in the individual studies, which required of several iterations between authors to reach a consensus.

\section{Limitations and strengths}

Firstly, despite of the large number of studies identified, we were able to meta-analyze only on a limited number of studies. The studies selected may not be representative. To mitigate this, we described the composition of the entire set of papers, showing that the two groups were fairly comparable in terms of study design, characteristics of the organizational interventions, case-mix of subjects, state of managed care (primary vs secondary prevention), and target outcomes (minor and major LEA).

Secondly, the seven meta-analyzed studies did not differentiate minor and major LEA at a patient level, making it difficult to draw clinical conclusions on the potential effectiveness of selected care. We conclude future studies should analyze specific interventions at an individual patient level. This should consider the possibility of combinations of different amputations, which can be more easily identified in longitudinal cohort analyses based on EMRs extracted from routine databases.

Thirdly, the strength of any interpretation is limited by the heterogeneity found even among the seven meta-analyzed studies. We found a general lack of standardization in the definition and comparability of the interventions under study. However, with regards to the validity of the comparisons tested in the meta-analysis, at the level of each study, we may assume that there was a fair comparison between alternative care arrangements. This allows drawing valid conclusions considering health system features only, and not the broader determinants of disease [46] which we know are important [47].

Lastly, concerning publication bias, results for total LEA include small studies with inadequate sample sizes where not enough LEA cases were reported to disaggregate results in terms of minor-major LEA. However, results for the major LEA subgroup are of a larger positive magnitude and include only studies of adequate quality, with no suggestion of overestimated results or publication bias, bringing certainty to our conclusions.

\section{Conclusion}

We identified that specific healthcare arrangements, which as main elements include care pathways, dedicated and multidisciplinary teams, and combined interventions, have the potential to cut the rate of major amputations in people with T2D and DFU in half.

Studies were heterogeneous in terms of design and definition of care arrangement, making it difficult to apply in context. Future research should focus on more comparable study designs, well-defined organizational targets, and a higher 
standardization or definition of care arrangements. This can be enabled through a better use of EMRs for cohort studies.

Reorganizing and optimising care arrangements and the use of a multidisciplinary approach are associated with improved outcomes for high-risk people with diabetes and foot ulceration. Future studies with more specific information on interventions are required to turn evidence into action, by tailoring care settings to the specific needs of patients that can benefit more.

Supplementary Information The online version contains supplementary material available at https://doi.org/10.1007/s00592-020-01662-x.

Authors' contributions All authors contributed to this study as according to the Journal's guidelines.

Funding This study was carried out with the financial support of the Marie Skłodowska-Curie Innovative Training Network (HealthProsHealthcare Performance Intelligence Professionals) with funding from the European Union Research Framework Programme Horizon 2020, under Grant Agreement No. 765141.

Code availability All statistical analyses were performed using the statistical package R v.1.2 and the metafor package. All R code is available at request.

\section{Compliance with ethical standards}

Conflict of interest Dr. Heiss reports research grants from the University of Surrey and the Economic and Social Research Council (ES/ V00980X/1) outside the submitted work.

Human and Animal Rights disclosure This article does not contain any studies with human or animal subjects performed by the any of the authors.

Informed consent No identifying information from individual patients was retrieved or published at any stage by any of the authors.

Availability of data and material Evaluated studies are publicly available peer-reviewed scientific publications.

Open Access This article is licensed under a Creative Commons Attribution 4.0 International License, which permits use, sharing, adaptation, distribution and reproduction in any medium or format, as long as you give appropriate credit to the original author(s) and the source, provide a link to the Creative Commons licence, and indicate if changes were made. The images or other third party material in this article are included in the article's Creative Commons licence, unless indicated otherwise in a credit line to the material. If material is not included in the article's Creative Commons licence and your intended use is not permitted by statutory regulation or exceeds the permitted use, you will need to obtain permission directly from the copyright holder. To view a copy of this licence, visit http://creativecommons.org/licenses/by/4.0/.

\section{References}

1. Carinci $\mathrm{F}$ et al (2015) Towards actionable international comparisons of health system performance: expert revision of the
OECD framework and quality indicators. Int J Qual Heal Care 27:137-146

2. Carinci F, Uccioli L, Massi Benedetti M, Klazinga NS (2019) An in-depth assessment of diabetes-related lower extremity amputation rates 2000-2013 delivered by twenty-one countries for the data collection 2015 of the Organization for Economic Cooperation and Development (OECD). Acta Diabetol. https:// doi.org/10.1007/s00592-019-01423-5

3. Harding JL, Pavkov ME, Gregg EW, Burrows NR (2019) Trends of nontraumatic lower extremity amputation in end-stage renal disease and diabetes, United States, 2000-2015. Diabetes Care 2:190296. https://doi.org/10.2337/dc19-0296

4. Armstrong DG, Boulton AJM, Bus SA (2017) Diabetic foot ulcers and their recurrence. N Engl J Med 376:2367-2375

5. Jeffcoate WJ, Vileikyte L, Boyko EJ, Armstrong DG, Boulton AJM (2018) Current challenges and opportunities in the prevention and management of diabetic foot ulcers. Diabetes Care 41:645-652

6. Rice JB et al (2014) Burden of diabetic foot ulcers for medicare and private insurers. Diabetes Care 37:651-658

7. Holman N, Young RJ, Jeffcoate WJ (2012) Variation in the recorded incidence of amputation of the lower limb in England. Diabetologia 55:1919-1925

8. Margolis DJ et al (2011) Location, location, location: geographic clustering of lower-extremity amputation among Medicare beneficiaries with diabetes. Diabetes Care 34:2363-2367

9. Carinci F, Massi Benedetti M, Klazinga NS, Uccioli L (2016) Lower extremity amputation rates in people with diabetes as an indicator of health systems performance A critical appraisal of the data collection 2000-2011 by the Organization for Economic Cooperation and Development (OECD). Acta Diabetol 53:825-832

10. Donabedian A (1966) Evaluating the quality of medical care. Milbank Mem Fund Q 44(Suppl):166-206

11. TRIAD Study Group (2010) T.T.S. Health systems, patients factors, and quality of care for diabetes: a synthesis of findings from the TRIAD study. Diabetes Care 33:940-947

12. Renders $C M$ et al (2001) Interventions to improve the management of diabetes in primary care, outpatient, and community settings. Diabetes Care 24:1821-1833

13. NICE (2019) NICE pathways diabetes. NICE pathways diabetes. https://pathways.nice.org.uk/pathways/diabetes.

14. Waddingham S (2019) Nine processes of care for diabetes. www.dtu.ox.ac.uk/riskengine. Accessed 5th June 2019.

15. NICE. 1 recommendationsldiabetic foot problems: prevention and management|GuidancelNICE

16. (ICHOM), I. C. for H. O. M. (2018) Type 1 and Type 2 diabetes in adults data collection reference guide

17. Schaper C et al (2019) IWGDF practical guidelines on the prevention and management of diabetic foot disease

18. Nuti S, Bini B, Ruggieri TG, Piaggesi A, Ricci L (2016) Bridging the gap between theory and practice in integrated care: the case of the diabetic foot pathway in Tuscany. Int J Integr Care $16: 9$

19. Moher D, Liberati A, Tetzlaff J, Altman DG (2009) Preferred reporting items for systematic reviews and meta-analyses: the PRISMA statement. PLoS Med 6:e1000097

20. Higgins J, Green S (eds) (2011) Cochrane handbook for systematic reviews of interventions version 5.1.0 [updated March 2011]

21. Wells GA, Shea B, O'Connell D, Peterson J, Welch V, Losos M et al (2019) The Newcastle-Ottawa Scale (NOS) for assessing the quality of nonrandomised studies in meta-analyses

22. Higgins JPT et al (2011) The Cochrane Collaboration tool for assessing risk of bias in randomised trials. BMJ 343:d5928 
23. Computing, R. F. for S. R Core Team (2013) R: a language and environment for statistical computing

24. Viechtbauer W (2010) Conducting meta-analyses in R with the metafor package. J Stat Softw 36:1-48

25. Crane M, Werber B (1999) Critical pathway approach to diabetic pedal infections in a multidisciplinary setting. J Foot Ankle Surg 38:30-33

26. Martínez-Gómez D et al (2014) Reduction in diabetic amputations over 15 years in a defined Spain population. Benefits of a critical pathway approach and multidisciplinary team work. Rev Esp Quimioter 27:170-179

27. Riaz M et al (2019) Impact of multidisciplinary foot care team on outcome of diabetic foot ulcer in term of lower extremity amputation at a tertiary care unit in Karachi, Pakistan. Int Wound $\mathbf{J}$ $16: 768-772$

28. Wrobel JS et al (2003) The relationship between provider coordination and diabetes-related foot outcomes. Diabetes Care 26:3042-3047

29. Nather A et al (2010) Value of team approach combined with clinical pathway for diabetic foot problems: a clinical evaluation. Diabet Foot Ankle 1:2000-2005

30. Liang R, Dai X, Zuojie L, Zhou A, Meijuan C (2012) Two-year foot care program for minority patients with type 2 diabetes mellitus of Zhuang Tribe in Guangxi, China. Can J Diabetes 36:15-18

31. McMurray SD, Johnson G, Davis S, McDougall K (2002) Diabetes education and care management significantly improve patient outcomes in the dialysis unit. Am J Kidney Dis 40:566-575

32. Frank U, Nikol S, Belch J et al (2019) ESVM Guideline on peripheral arterial disease. Vasa - Eur J Vasc Med 48:1-79

33. Rydén L et al (2013) ESC Guidelines on diabetes, pre-diabetes, and cardiovascular diseases developed in collaboration with the EASD: the Task Force on diabetes, pre-diabetes, and cardiovascular diseases of the European Society of Cardiology (ESC) and developed in collaborati. Eur Heart J 34:3035-3087

34. Tan T, Shaw EJ, Siddiqui F, Kandaswamy P, Barry PW, et al (2011) Inpatient management of diabetic foot problems: summary of NICE guidance. BMJ Open 342

35. Heiss $\mathrm{C}$ et al (2019) The compelling arguments for the need of medical vascular physicians in Europe. Vasa Eur J Vasc Med $48: 1-5$
36. Kodner DL (2009) All together now: a conceptual exploration of integrated care. Healthc Q 1:6-15

37. Valentijn PP, Schepman SM, Opheij W, Bruijnzeels MA (2013) Understanding integrated care: a comprehensive conceptual framework based on the integrative functions of primary care. Int J Integr Care 13:e010-e010

38. Shortell SM, Gillies RR, Anderson DA (1994) The new world of managed care: creating organized delivery systems. Health Aff (Millwood) 13:46-64

39. Driver VR, Fabbi M, Lavery LA, Gibbons G (2010) The costs of diabetic foot: the economic case for the limb salvage team. J Vasc Surg 52:17S-22S

40. Kerr M et al (2019) The cost of diabetic foot ulcers and amputations to the National Health Service in England. Diabet Med 36:995-1002

41. NHS Digital (2017) National Diabetes Foot Care Audit (NDFA) 2014-2016

42. Schuivens PME et al (2020) Impact of the COVID-19 lockdown strategy on vascular surgery practice: more major amputations than usual. Ann Vasc Surg 69:74-79

43. Bornstein SR et al (2020) Practical recommendations for the management of diabetes in patients with COVID-19. Lancet Diabetes Endocrinol 8:546-550

44. Correa A et al (2016) Royal College of General Practitioners Research and Surveillance Centre (RCGP RSC) sentinel network: a cohort profile. BMJ Open 6:e011092

45. Cunningham SG et al (2016) Core Standards of the EUBIROD Project. Defining a European diabetes data dictionary for clinical audit and healthcare delivery. Methods Inf Med 55:166-176

46. Marmot M (2005) Social determinants of health inequalities. Lancet 365:1099-1104

47. Whyte MB et al (2019) Disparities in glycaemic control, monitoring, and treatment of type 2 diabetes in England: a retrospective cohort analysis. PLOS Med 16:e1002942

Publisher's Note Springer Nature remains neutral with regard to jurisdictional claims in published maps and institutional affiliations. 